\title{
AG490 suppresses EPO-mediated activation of JAK2-STAT but enhances blood flow recovery in rats with critical limb ischemia
}

\author{
Han-Tan Chai ${ }^{1 \dagger}$, Hon-Kan Yip ${ }^{1,2,3,4,5+}$, Cheuk-Kwan Sun ${ }^{6}$, Shu-Yuan Hsu ${ }^{2,7}$ and Steve Leu ${ }^{2 *}$
}

\begin{abstract}
Background: Erythropoietin (EPO) has been demonstrated to enhance recovery in ischemic organs through enhancing angiogenesis. In this study, we used an experimental critical limb ischemia (CLI) rat model to reveal the underlying mechanisms and directly examine the benefits of the anti-apoptotic capacity of EPO in the acute phase of limb ischemia and following blood flow recovery.

Methods: To determine the role of the JAK2/STAT pathway in EPO-enhanced recovery after CLI, male Sprague-Dawley rats ( $n=8$ for each group) were divided into group 1 (normal control), group 2 (CLI treated with normal saline), group 3 (CLI treated with EPO), group 4 (CLI treated with AG490, a JAK2 inhibitor), and group 5 (CLI treated with EPO and AG490). Animals were sacrificed either at day 1 or day 14 and biochemical and histopathological examination of ischemic quadriceps were conducted.

Results: At day 1, EPO administration reduced expression levels of apoptotic indices and activated the JAK2/STAT pathway; this activation was inhibited by additional AG490 treatment. Furthermore, the decrease in the size of the infarcted area, as well as activation of ERK1/2 and JNK showed similar regulatory trends with EPO or AG490 treatment. Of Interest, EPO and AG490 in combination showed a synergistic effect, increasing expression levels of antioxidants (GR, GPx, NQO-1) and decreasing transcriptional levels of pro-inflammatory factors (TNF-a, NF-kB). At day 14, laser Doppler analysis showed that the blood flow recovery was enhanced by EPO, AG490, or combined treatment.

Conclusion: Although inhibition of the JAK2/STAT pathways reduces the anti-apoptotic effects of EPO in the early phase of CLI, the benefits of AG490 in anti-inflammation and anti-oxidation still play a positive role in enhancing blood flow recovery after $\mathrm{CLI}$.
\end{abstract}

Keyword: Erythropoietin, AG490, JAK2, Critical limb ischemia, Apoptosis

\section{Background}

Peripheral arterial disease (PAD) is caused by chronic inflammatory processes associated with atherosclerosis [1]. Critical limb ischemia (CLI), which results in significant blood flow reduction in feet and hands, is the most severe form of PAD [2]. Although endovascular intervention and open surgical techniques are widely used treatments for CLI, amputation remains the final option for a certain subset of patients [3-5]. Under general medical care conditions, one year after diagnosis of CLI,

\footnotetext{
* Correspondence: leu@mail.cgu.edu.tw

†Equal contributors

${ }^{2}$ Institute for Translational Research in Biomedicine, Kaohsiung Chang Gung

Memorial Hospital, Kaohsiung, Taiwan

Full list of author information is available at the end of the article
}

half of patients are dead or alive with amputations, while only quarter of patients see symptoms resolve [2]. So far, there is no satisfying pharmacologic therapy to efficiently reverse arterial occlusive lesions, or the subsequent impaired perfusion in ischemic limbs of patients [6]. The purpose of pharmacologic treatment for CLI includes risk factor modification and efforts to improve blood flow $[7,8]$. However, only patients with mild to moderate intermittent claudication are advised to undergo pharmacologic therapy [7]. Therefore, alternate treatment approaches are urgently needed for CLI.

Erythropoietin (EPO), a $165 \mathrm{kDa}$ secreted glycoprotein, was first characterized as a hematopoietic factor and has 
been widely used for the clinical treatment of anemia [9-11]. EPO not only promotes the proliferation and differentiation of erythroid precursors, but also plays an important role as an anti-apoptotic factor for hematopoietic cells [12]. In general, the expression level of erythropoietin is upregulated under hypoxic conditions and mediated by a transcription factor, hypoxia inducible factor-1 (HIF-1) $[13,14]$. EPO is mainly produced by cells of the peritubular capillary endothelium of the kidney [15], while EPO receptors (EPOR) are widely expressed in various tissues, including brain, retina, heart, kidney, smooth muscle, myocardium, and endothelium [15]. The EPO-mediated protective responses in anti-apoptosis are also found in non-hematopoietic cells, e.g., renal tubular cells [16], neurons [17], retina cells [18], cardiomyocytes [19], and endothelial cells [17]. Recent studies also demonstrated that EPO plays multiple functional roles in anti-inflammation $[15,20]$, angiogenesis $[21,22]$, and in endothelial response to increasing nitric oxide production [23]. The therapeutic efficacy of EPO in amelioration of organ ischemic injury or ischemia-reperfusion injury has been evaluated through experimental animal models as well as clinical applications [24-28].

EPO is activated through its binding to the EPO receptor (EPOR), which is composed of two identical subunits $[29,30]$. After binding, the receptor is dimerized and Janus kinase-2 (JAK2) is then recruited to the receptor complex $[29,30]$. After binding of EPO and EPOR, several substrates of JAK2, including transcription factor signal transducer and activator of transcription (STAT) are recruited to the docking site of $\operatorname{EPOR}[29,30]$. STATs are phosphorylated by JAK kinases, leading to dimerization and subsequent translocation to the nucleus [29, 30]. After nuclear translocation, STATs bind to promoters of several genes involved in anti-apoptosis, including $\mathrm{Bcl}-\mathrm{xL}, \mathrm{Bcl}-2$ and $\mathrm{c}-\mathrm{Myc}$. However, although the activation of JAK2/STATs plays an anti-apoptotic role in organ injury, this activated signaling is also involved in upregulation of pro-inflammatory cytokine generation [31-33]. Inhibition of JAK2 activity through its inhibitors (i.e., AG490) has been applied as an approach to treat ischemia-reperfusion injury and autoimmune arthritis in animal models [34, 35]. Hence, revealing the underlying mechanism of EPO-mediated cellular response is important for the selection and adjustment of clinical application of EPO in different types of organ injuries.

Although EPO has been demonstrated to have therapeutic efficacy in treating critical limb ischemia in experimental animal models [22], the underlying mechanisms are still not completely clear. In the present study, we applied rat CLI models with a JAK2 inhibitor to determine the whether the JAK2/STAT pathway is essential for EPOmediated blood flow recovery after CLI. In addition, whether the anti-inflammatory role of AG490 contributes in injury recovery from CLI was also examined.

\section{Methods \\ Animal model of critical limb ischemia}

Pathogen-free, adult male Sprague-Dawley (SD) rats $(n=60)$ weighing 320-350 g (Charles River Technology, BioLASCO Taiwan Co. Ltd., Taiwan) were used in this study ( $n=16$ for each group): Group 1, normal control; Group 2, critical limb ischemia (CLI) with normal saline; Group 3, CLI with EPO; Group 4, CLI with AG490; Group 5, CLI with EPO and AG490. EPO was injected intramuscularly $(1000 \mathrm{IU} / \mathrm{kg})$, while AG490 was injected intraperitoneally $(3 \mathrm{mg} / \mathrm{kg})$. EPO and AG490 were administrated at $30 \mathrm{~min}, 24 \mathrm{~h}$, and $48 \mathrm{~h}$ after induction of CLI. Animals were sacrificed at either day 1 or day 14 (8 animals from each group were sacrificed at each time-point). For induction of critical limb ischemia by ligation of the femoral artery, rats were placed in a supine position on a warming pad at $37{ }^{\circ} \mathrm{C}$ with the left hind limbs shaved. Under sterile conditions, the left femoral artery, small arterioles and circumferential femoral artery were exposed and ligated over their proximal and distal portions before removal. To avoid the presence of collateral circulation, the branches were removed together. After sacrifice, the left quadricep muscles were collected for individual study.

\section{Measurement of blood flow with laser doppler}

Rats were anesthetized by inhalation of $2.0 \%$ isoflurane prior to CLI induction and on days 2 and 14 after CLI induction prior to being sacrificed ( $n=8$ for each group). The rats were placed in a supine position on a warming pad at $37{ }^{\circ} \mathrm{C}$. After being shaved over bilateral hind limbs and inguinal areas, blood flow was surveyed by a Laser Doppler scanner (moorLDLS, Moor, UK). The ratio of blood flow in the left hind limb (ischemic) to that in the right side (normal) was applied to determine the blood flow recovery after CLI.

\section{Quantitative reverse transcription-polymerase chain reaction}

Quantitative mRNA levels were determined using realtime reverse transcription-polymerase chain reaction (RTPCR) with the Applied Biosystems 7900 HT Sequence Detection System (Applied Biosystems) and TaqMan Gene Expression Assay as previously described [36].

\section{Western blot analysis}

Equal amounts $(10-30 \mu \mathrm{g})$ of protein extracts from ischemic quadriceps of the animals ( $n=6$ for each group) were loaded and separated by SDS-PAGE using 7 or $12 \%$ acrylamide gradients. Proteins were then transferred to nitrocellulose membranes. The membranes were incubated with monoclonal antibodies against 
JAK2 (1:1000, Abcam), phosphorylated JAK2 (1:1000, Abcam), STAT1 (1: 1000, Cell Signaling), phosphorylated STAT1 (1: 1000, Cell Signaling), STAT3 (1:500, Cell Signaling), phosphorylated STAT3 (1:1000, Cell Signaling), STAT5 (1:1000, Abcam), phosphorylated STAT5 (1:500, Abcam), Akt (1:1000, Cell Signaling), phosphorylated Akt (1:2000, Cell Signaling), JNK (1:500, Sigma), phosphorylated JNK (1:1000, Abcam), ERK1/2 (1:1000, Cell Signaling), and phosphorylated ERK1/2 (1:2000). Signals were detected with HRP-conjugated goat anti-mouse or goat anti-rabbit IgG. Immunoreactive bands were visualized by enhanced chemiluminescence (ECL; Amersham Biosciences) which was then exposed to Biomax L film (Kodak). For quantification, ECL signals were digitized using Labwork software (UVP).

\section{Histopathological and immunostaining}

The immunofluorescence (IF) staining and immunohistochemical (IHC) staining were performed as previously described [37]. In brief, fixed cryosections $(10 \mu \mathrm{m})$ of quadriceps were incubated with antibodies against CD31 (1:200, Abcam), EPOR (1:500, Abcam), or $\alpha$-SMA (1:400, Millipore) at $4{ }^{\circ} \mathrm{C}$ overnight, followed by incubation with fluorescence or HRP-conjugated secondary antibodies. For quantification, ten randomly selected HPFs (high power fields, 200x) were analyzed in each section. The mean number per HPF for each animal was then determined by summation of all numbers divided by 30 .

\section{Statistical analysis}

Data was expressed as mean values (mean $\pm \mathrm{SD}$ ). The significance of differences between two groups was evaluated with $t$-test. The significance of differences among groups was evaluated using one-way ANOVA, followed by Bonferroni multiple comparison post hoc test. Statistical analysis was performed using Prism 5 statistical software (GraphPad Software, La Jolla, CA, USA). A probability value of less than 0.05 was considered statistically significant.

\section{Results}

\section{AG490 administration inhibits phosphorylation of STATs} in quadriceps

To clarify the activation of the JAK2 pathway in EPOmediated enhanced recovery from limb ischemia, AG490, a well-known JAK2 inhibitor, was applied to rats that underwent femoral artery ligation. Apoptosis occurs in the acute phase of ischemia. Since EPO has been reported to function in the inhibition of apoptosis, the quadriceps were isolated to examine the activation of JAK2 and its downstream STATs in the ischemic area (Fig. 1a). Results showed that both total and phosphorylated JAK2 was significantly higher in CLI rats with EPO administration (Fig. 1b and c). The increased JAK2 phosphorylation in quadriceps of EPO-treated CLI rats was blocked by AG490 (Fig. 1c). To further confirm the activation of the JAK2 pathway in CLI rats with EPO treatment, the expression levels and phosphorylation status of STAT family proteins in ischemic quadriceps were then examined (Fig. 1a). Along with the activation of EPO, the expression levels of phosphorylated STAT1 (Fig. 1e) and STAT5 (Fig. 1i) were increased in quadriceps of CLI rats with EPO treatment. The EPOmediated activation of the STATs was also abolished with AG490. Of interest, the total expression and phosphorylated levels of STAT3 were significantly increased by induction of CLI. These increases of STAT3 levels were not further enhanced by EPO treatment, but were abolished by AG490 (Fig. 1f and g).

\section{EPO administration showed an anti-apoptotic activity in ischemic quadriceps}

Although EPO has been found to have an anti-apoptotic function in vitro in cultured endothelial cells [38], its physiological anti-apoptotic role has not been confirmed in vivo. Hence, TUNEL assays were performed in situ to detect the apoptotic nuclei in ischemic quadriceps (Fig. 2a-f). Results showed that the number of apoptotic nuclei was increased after CLI induction (Fig. 2b) and this increase was reversed with EPO treatment (Fig. 2c). The administration of AG490 further increased the number of apoptotic nuclei in CLI rats (Fig. 2d). Moreover, reduction of the number of apoptotic nuclei in quadriceps of CLI rats by EPO was blocked by additional AG490 treatment (Fig. 2e).

Quantitated real-time reverse transcription polymerase chain reaction (RT-qPCR) was also performed to confirm the results of TUNEL assay (Fig. 2g-i). RT-qPCR examination of transcripts of Bax (Fig. $2 \mathrm{~h}$ ) and caspase-3 (Fig. 2i), two pro-apoptotic indices showed similar regulatory trends to those observed in the TUNEL assay. In contrast, mRNA expression levels of Bcl-2, an antiapoptotic factor, were decreased after CLI induction and reverted with EPO treatment (Fig. 2g). In addition, the blocking of EPO-mediated cellular protective function against apoptosis in ischemic quadriceps by AG490 was confirmed by RT-qPCR (Fig. 2g-i).

\section{Infarct area in ischemic quadriceps is reduced by EPO treatment}

After CLI induction, the infarction of skeletal muscles is usually accompanied by with loss of blood flow and cellular apoptosis. Following the examination of apoptotic events at the tissue and molecular levels, histopathological examination was performed to determine the infarcted area in the ischemic quadriceps (Fig. 3). Quadriceps from rats were isolated and used for 


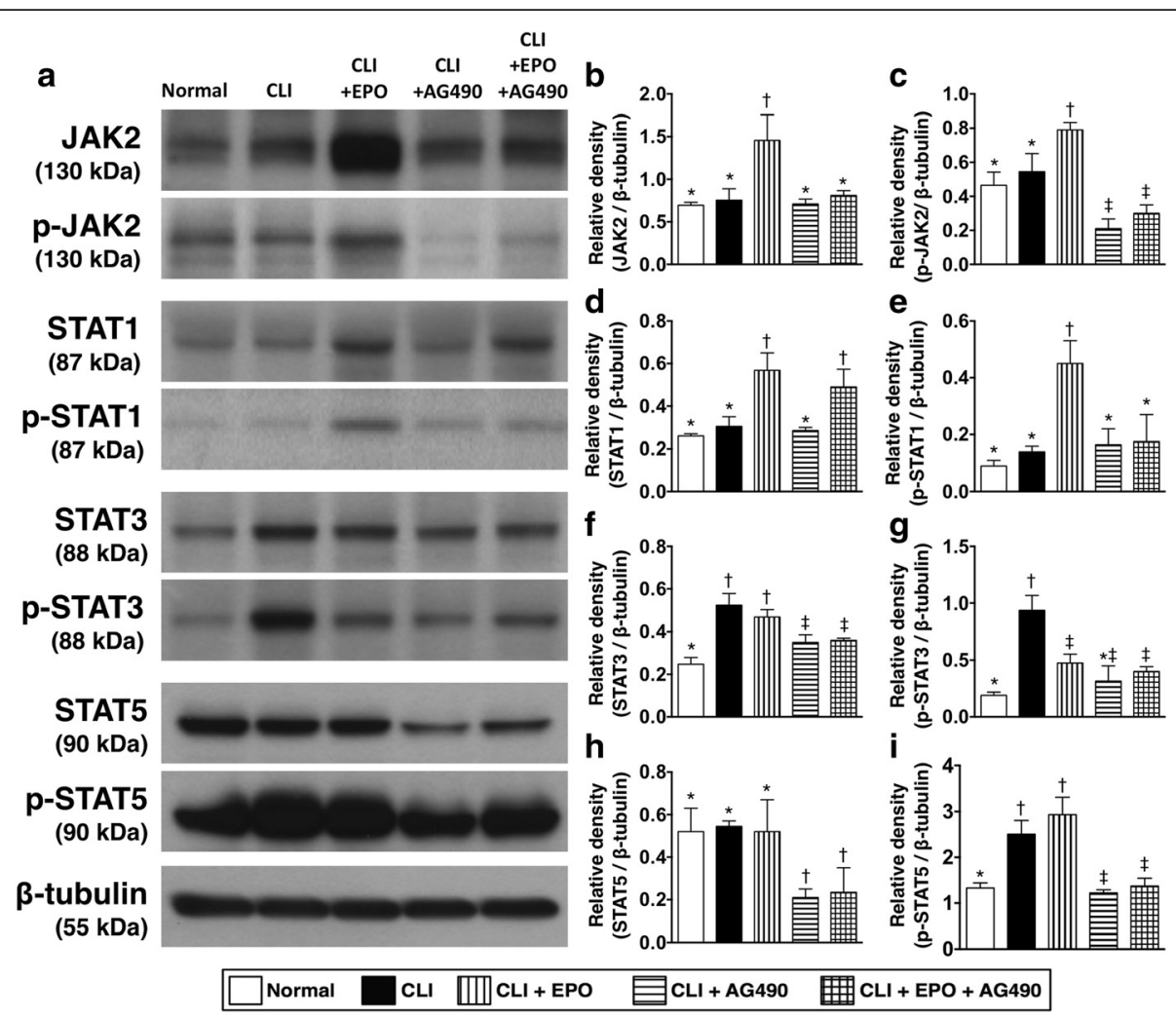

Fig. 1 EPO regulates the expression and phosphorylation levels of JAK/STATs in quadriceps after critical limb ischemia. a Twenty-four hours after CLI, total protein was extracted from quadriceps and Western blots were performed with antibodies against JAK2, phospho-JAK2, STAT5, phospho-STAT5, STAT1, phospho-STAT1, STAT3, and phospho-STAT3. $\mathbf{b}$ and $\mathbf{c}$ The expression levels of total and phosphorylated JAK2. $\mathbf{d}$ and $\mathbf{e}$ The expression levels of total and phosphorylated STAT1. $\mathbf{f}$ and $\mathbf{g}$ The expression levels of total and phosphorylated STAT3. $\mathbf{h}$ and $\mathbf{i}$ The expression levels of total and phosphorylated STAT5. Statistical analysis used one-way ANOVA followed by Bonferroni multiple comparison post hoc test ( $n=8$ for each group). Symbols $\left({ }^{*},+, \neq,\right)$ indicate significance at $p$ value less than 0.05. EPO, Erythropoietin; JAK, Janus kinase; STAT, Signal Transducer and Activator of Transcription

preparing cryo-section followed by Hematoxylin and Eosin (H\&E) staining. Infarcted areas in the quadriceps of all groups were measured and calculated. The results showed that the infarct areas increased after CLI induction and that this increase was reverted with EPO treatment (Fig. 3b and c). AG490-only treatment increased the infarct area in the quadriceps of CLI rats (Fig. 3d), and reduction of the infarcted area in the ischemic quadriceps by EPO was inhibited by additional treatment with AG490 (Fig. 3e).

EPO and AG490 contribute to anti-inflammation and antioxidation in the ischemic quadriceps

After ischemic injury, oxidative stress and inflammation usually impair tissue regeneration and micro-circulation reconstruction. Hence, the regulation of expression levels of inflammatory and anti-oxidative factors by EPO and AG490 in ischemic quadriceps was examined. Twenty-four hours after CLI induction, the transcriptional levels of inflammatory and anti-inflammatory factors were examined using RT-qPCR (Fig. 4). Results showed that gene expression levels of tumor necrosis factor (TNF)- $\alpha$ and nuclear factor kappa-light-chain-enhancer of activated B cells (NF-kB), two inflammatory factors, were increased after CLI induction, while both EPO and AG490 reduced their expression levels in ischemic quadriceps (Fig. 4a and b). It is worth noting that, instead of inhibition, combined AG490 and EPO treatment showed a synergistic effect in reducing the expression levels of inflammatory cytokines. The transcriptional levels of interleukin (IL)-10, an anti-inflammatory cytokine, was reduced after CLI induction; whereas EPO, AG490, or combined EPO and AG490 treatments increased the expression levels of IL-10 in ischemic quadriceps with a synergistic pattern (Fig. 4c).

The mRNA expression levels of anti-oxidant genes, heme oxygenase (HO)-1, NAD(P)H:quinone oxidoreductase 1 (NQO1), glutathione reductase (GR), and glutathione peroxidase (GPx) were increased after CLI induction (Fig. 4d-g). Treatment with EPO, AG490, or combined EPO and AG490 all further increased the transcripts. Showing the same trend as IL-10, EPO and AG490 

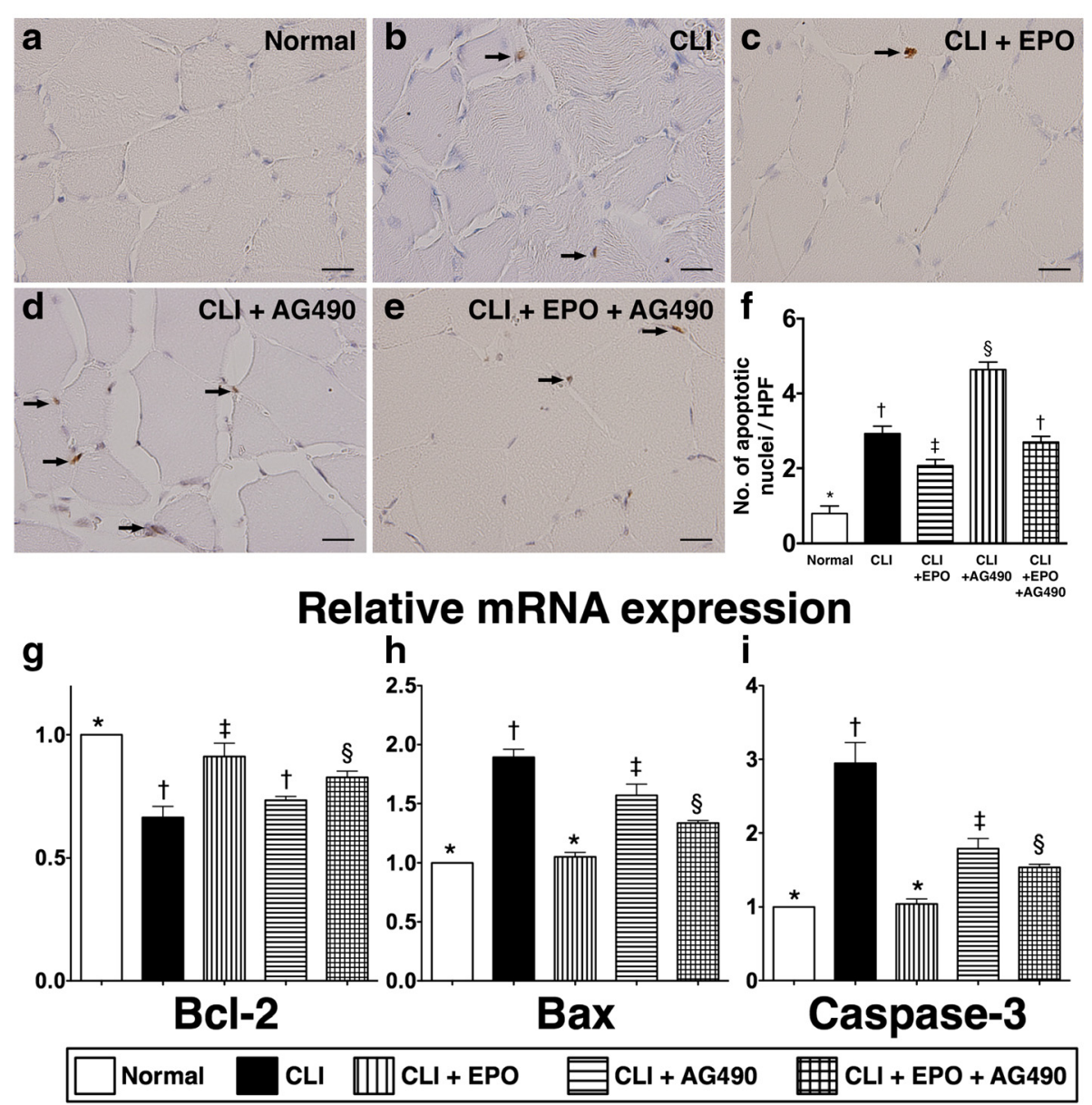

Fig. 2 EPO administration reduces the number of apoptotic nuclei and regulates transcriptional levels of apoptosis indices. Twenty-four hours after CLI, quadriceps from ischemic limbs were isolated for cryosections and total RNA extraction. Cryosections were used for terminal deoxynucleotidyl transferase dUTP nick end labeling (TUNEL) assay to detect apoptotic nuclei, and total RNA was used for real-time reverse transcription polymerase chain reaction (RT-qPCR) with specific primers to determine the mRNA expression levels of Bcl-2, Bax, and caspase-3. a-e The apoptotic nuclei were identified with TUNEL assay and indicated with black arrows. $\mathbf{f}$ The quantitation of the number the apoptotic nuclei. $\mathbf{g}$ The mRNA expression level of BCl-2, an anti-apoptotic index. $\mathbf{h}$ and $\mathbf{i}$ The mRNA expression levels of Bax and caspase-3, two apoptotic indices. Statistical analysis used one-way ANOVA followed by Bonferroni multiple comparison post hoc test ( $n=8$ for each group). Symbols $(*,+, \neq, \S)$ indicate significance at $p$ value less than 0.05

synergistically enhanced the expression levels of antioxidants (Fig. 4d-g).

\section{Phosphorylation of ERK $1 / 2$ with that activation of the JAK2/STAT pathway}

Although JAK2/STAT signaling is usually considered to be the downstream signal transduction of EPO, other kinase-based signals, including MEK/ERK and PI3K/Akt pathways have also been demonstrated to be involved in EPO-triggered intracellular signaling. Therefore, we next examined the total and phosphorylation levels of Akt, JNK, and ERR1/2 proteins in the ischemic quadriceps (Fig. 5a). Results showed that EPO, AG490, or combined EPO and AG490 treatment did not regulate the total expression level and phosphorylation levels of Akt after CLI induction (Fig. 5b and c). The total expression levels of JNK showed no difference among all groups (Fig. 5d), while the phosphorylation levels of JNK were increased by induction of CLI. However, EPO and AG490 did not regulate the activation of JNK (Fig. 5e) The total expression levels of ERK1/2 showed no difference among all groups (Fig. 5f), while the phosphorylation of ERK1/2 were increased after CLI induction and further enhanced with EPO treatment (Fig. 5g). However, AG490-only treatment or combined EPO and AG490 after CLI did not regulate the phosphorylation of ERK1/2.

\section{Activation of JAK2 signaling is important in EPO- mediated increased expression of EPO receptors}

The expression levels of EPO receptors in endothelial cells were examined through immunofluorescence staining in quadriceps (Fig. 6). Results showed that the 

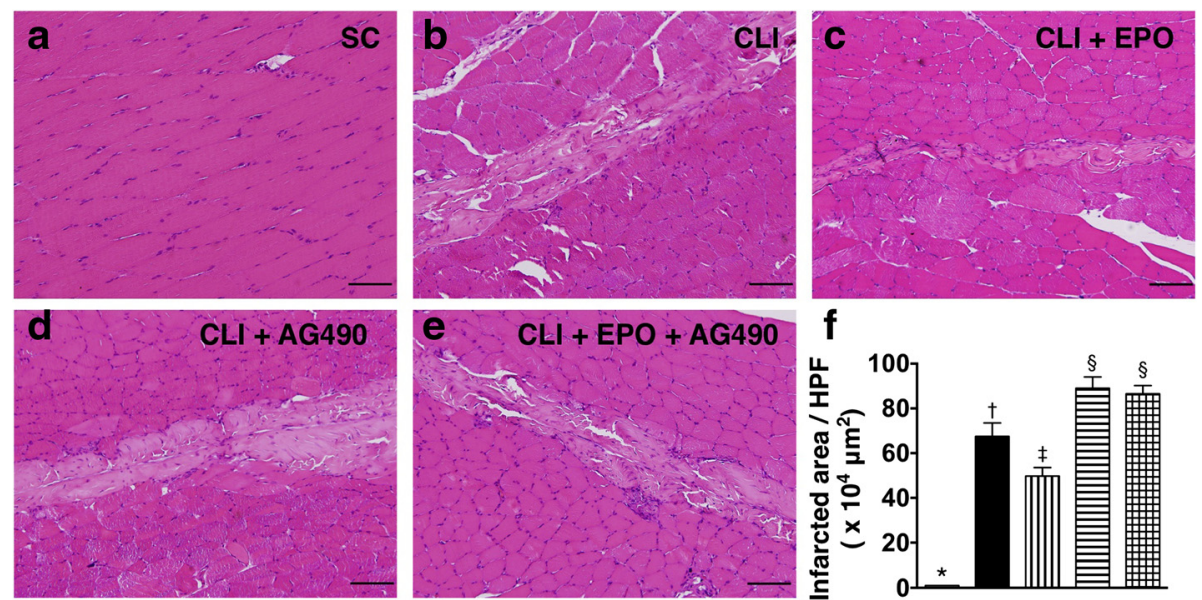

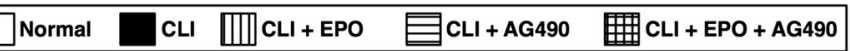

Fig. 3 EPO reduces the infarcted area in ischemic quadriceps. The infarcted area of the quadriceps was determined using Hematoxylin and Eosin (H\&E) staining. a Quadricep section from normal rats. b Quadricep section from rats with critical limb ischemia (CLI). c Quadricep section from CLI rats treated with EPO. $\mathbf{d}$ Quadricep section from CLI rats treated with AG490. e Quadricep section from CLI rats treated with EPO and AG490. f Quantitative evaluation of the infarct areas in quadriceps. Statistical analysis used one-way ANOVA followed by Bonferroni multiple comparison post hoc test ( $n=8$ for each group). Symbols $(*,+, \neq, \S)$ indicate significance at $p$ value less than 0.05
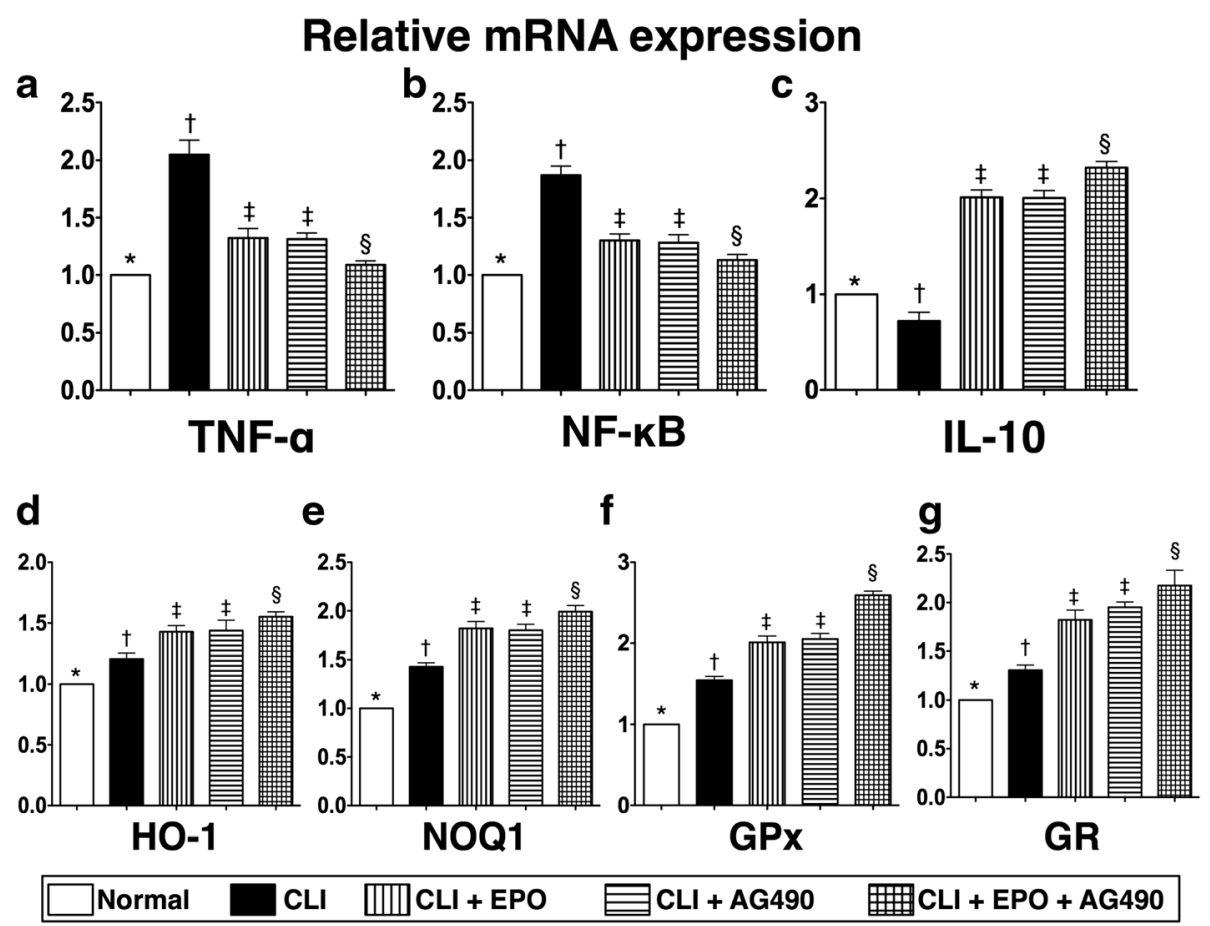

Fig. 4 EPO and AG490 administrations reduce the mRNA expression levels of inflammatory factors and increase the expression levels of antioxidants. Twenty-four hours after CLI, total RNA extracted from quadriceps was used for real-time reverse transcription polymerase chain reaction (RT-qPCR) to determine the mRNA expression levels of TNF-a, NF-kB, IL-10, HO-1, NQO-1, GPx and GR. a The mRNA expression levels of TNF-a. $\mathbf{b}$ The mRNA expression levels of NF-kB. $\mathbf{c}$ The mRNA expression levels of IL-10. $\mathbf{d}-\mathbf{g}$ The mRNA expression levels of HO-1, NQO-1, GPX, and $G R$, four antioxidants. Statistical analysis used one-way ANOVA followed by Bonferroni multiple comparison post hoc test ( $n=8$ for each group). Symbols $(*,+, \neq, \S)$ indicate significance at $p$ value less than 0.05 


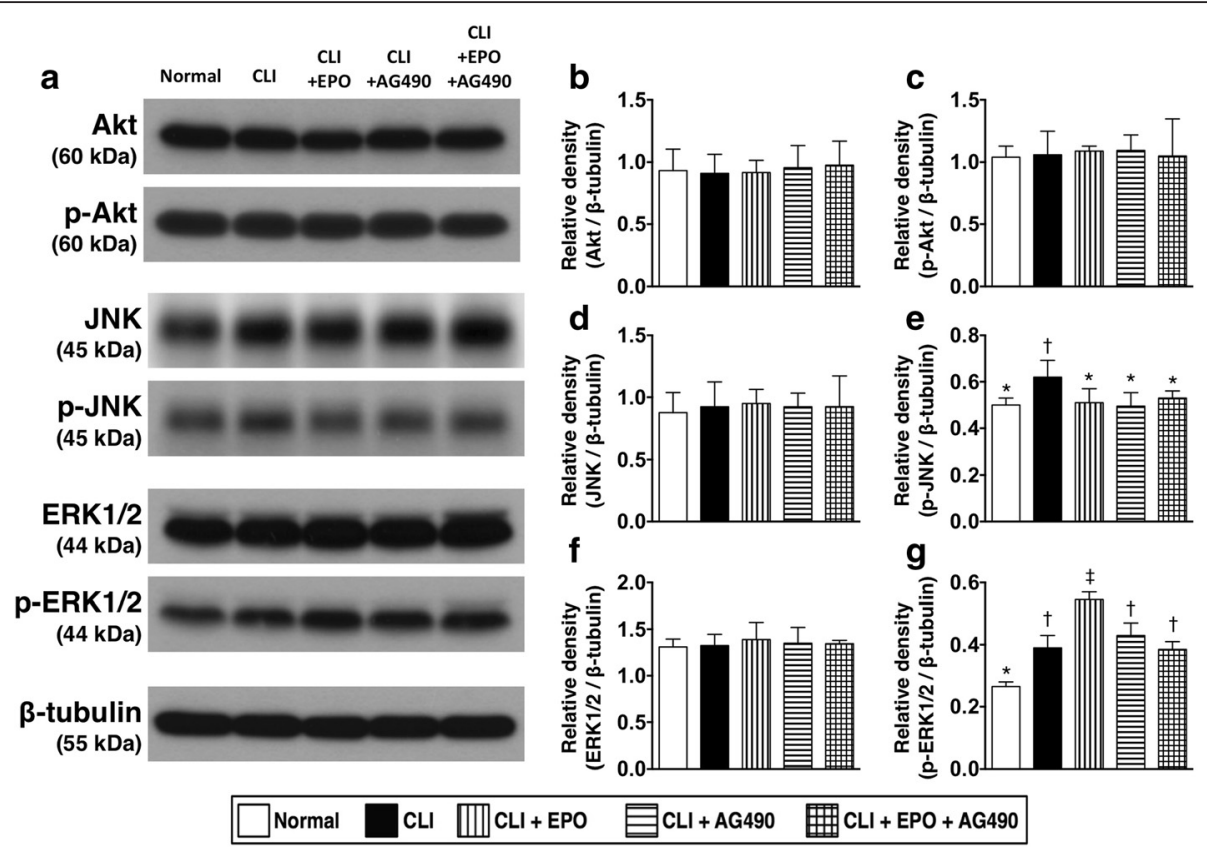

Fig. 5 Expression and phosphorylation levels of Akt, JNK, and ERK1/2 in ischemic quadriceps. a Total and phosphorylated protein expression levels of Akt, JNK, and ERK1/2 were examined through Western blots with specific antibodies. b Expression levels of total Akt. c Expression levels of phosphorylated Akt. $\mathbf{d}$ Expression levels of total JNK. e Expression levels of phosphorylated JNK. $\mathbf{f}$ Expression levels of total ERK1/2. $\mathbf{g}$ Expression levels of phosphorylated ERK1/2. The protein expression levels are quantitated and normalized with the expression levels of $\beta$-tubulin. Statistical analysis used one-way ANOVA followed by Bonferroni multiple comparison post hoc test ( $n=8$ for each group). Symbols $(*,+, \neq)$ indicate significance at $p$ value less than 0.05
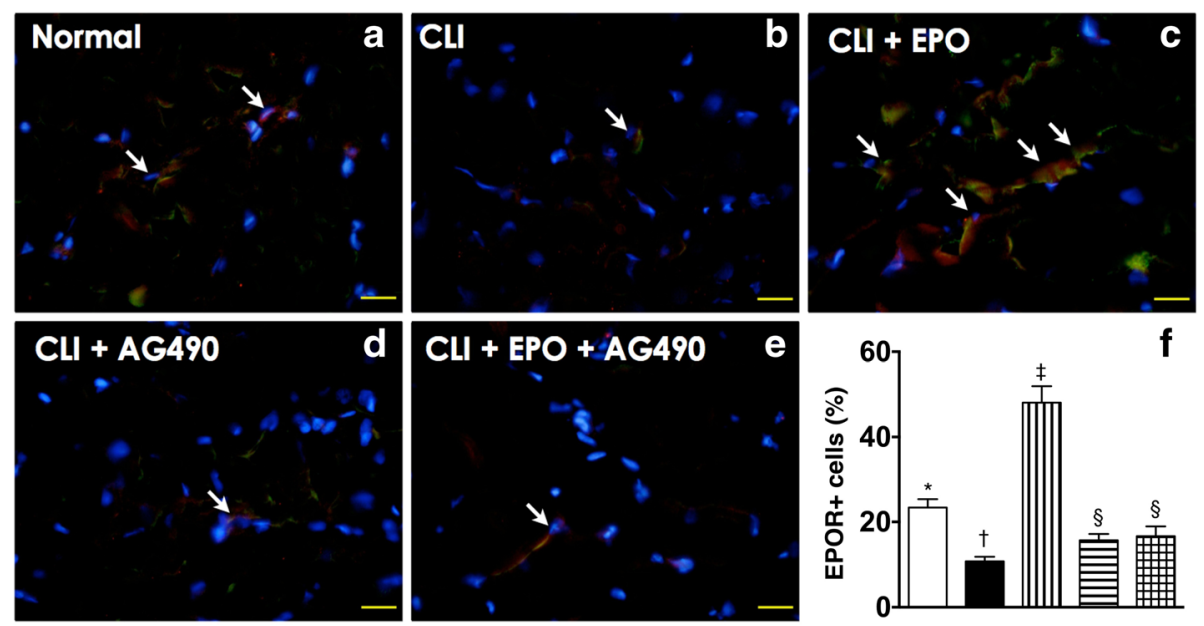

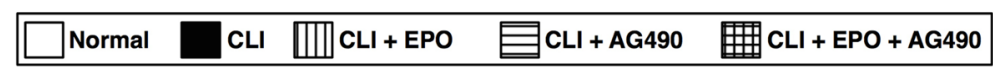

Fig. 6 EPO administration increases the expression levels of EPO receptors in ischemic limbs. Twenty-four hours after CLI, quadriceps from ischemic limbs were isolated for cryosections and used for immunohistochemical staining to detect the expression and distribution of EPO receptor (EPOR). a-e The endothelial cells were recognized with antibodies against CD31 and shown in green color. The expression of EPOR was recognized with antibodies against EPOR and shown in red color. Arrows indicated the localization of EPOR positively stained (EPOR+) cells in quadriceps. $\mathbf{f}$ The quantitation of the number of EPOR+ cells. Statistical analysis used one-way ANOVA followed by Bonferroni multiple comparison post hoc test $(n=8$ for each group). Symbols $(*,+, \neq, \S)$ indicate significance at $p$ value less than .05 
number of EPO positively stained $(\mathrm{EPO}+)$ endothelial cells was reduced after CLI induction, indicating the loss of endothelial cells in the acute phase after CLI induction (Fig. 6b). However, EPO treatment not only reverted the number of EPOR+ endothelial cells, but also increased the intensity of EPOR signals (Fig. 6c). Of importance, the increase of EPOR + cells by EPO was blocked by additional AG490 treatment (Fig. 6e).

\section{Enhanced blood flow recovery with EPO and AG490 treatment}

Fourteen days after CLI induction, we applied laser Doppler analysis to examine the blood flow recovery in the ischemic limbs. After quantitation, the ratio of ischemia to normal blood flow (INBF) was used as the parameter to determine the condition of blood flow recovery (Fig. 7). The ratio of INBF in the CLI only group was significantly lower than in the normal group by day 14 after CLI. It is interesting that not only EPO, but also AG490 enhanced the blood flow recovery after CLI. However, combined EPO and AG490 treatment did not show synergistic effects in enhancing blood flow recovery in ischemic limbs. To further confirm the enhanced blood flow recovery by EPO and AG490, immunohistochemical staining against $\alpha$-smooth muscle actin ( $\alpha$-SMA) was performed to determine the number of vessels in ischemic limbs (Fig. 8). As the result of laser Doppler examination, the numbers of small vessels (diameter $<35 \mu \mathrm{m})$ in the quadriceps was reduced by CLI induction and reverted with EPO treatment. AG490-only treatment also increased the number of small vessels; however, the increment was fewer than that in those treated with EPO. No synergistic effect was found in CLI rats receiving EPO and AG490 combined therapy.

\section{Discussion}

In the present study, we directly applied an experimental rat model with critical limb ischemia to evaluate the effects of EPO and JAK2 inhibitor AG490 in preventing apoptosis as well as in enhancing blood recovery in ischemic limbs. In addition, we also performed biochemical analysis, histopathological examination, and immunostaining to detect the activation of underlying signaling responding to CLI insults and EPO treatment. The results suggest that EPO can prevent cellular apoptosis and reduce the size of the infarct area in the ischemic quadriceps during the acute phase of CLI (Figs. 2 and 3). We also found that the phosphorylation of STAT1 and STAT5, but not STAT3, was activated by EPO-mediated JAK2 activation in the CLI areas (Fig. 1). Moreover, our results showed that the JAK2 inhibitor AG490 blocked the activation of JAK2/STAT signaling and abolished the antiapoptotic efficacy of EPO (Figs. 1-3). However, AG490only treatment increased the synthesis of anti-oxidants

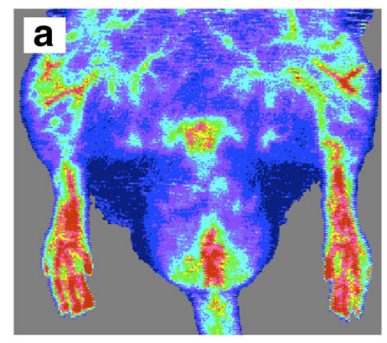

Normal

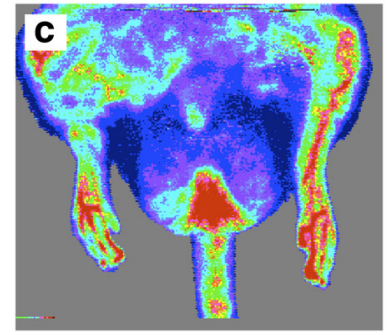

CLI + EPO

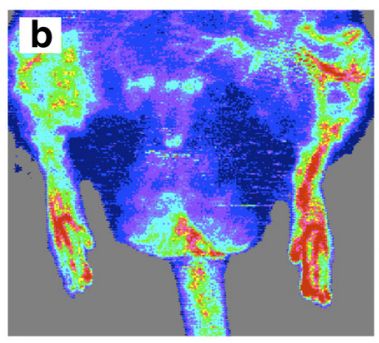

CLI
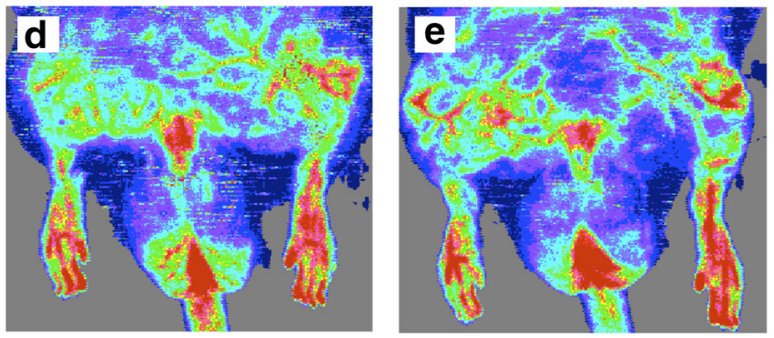

CLI + AG490 CLI + EPO + AG490

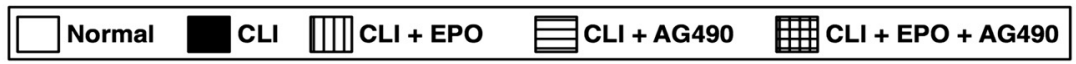

Fig. 7 EPO and AG490 administrations increase the blood flow recovery after critical limb ischemia induction in rats. a-e Fourteen days after CLI, blood flows in the rat lower limbs were measured by laser Doppler. f Quantitation and calculation of blood flows. Statistical analysis using one-way ANOVA, followed by Bonferroni multiple comparison post hoc test ( $n=8$ for each group). Symbols $\left({ }^{*},+, \neq\right)$ indicate significance at $p$ value less than 0.05. INBF, ischemia to normal blood flow 

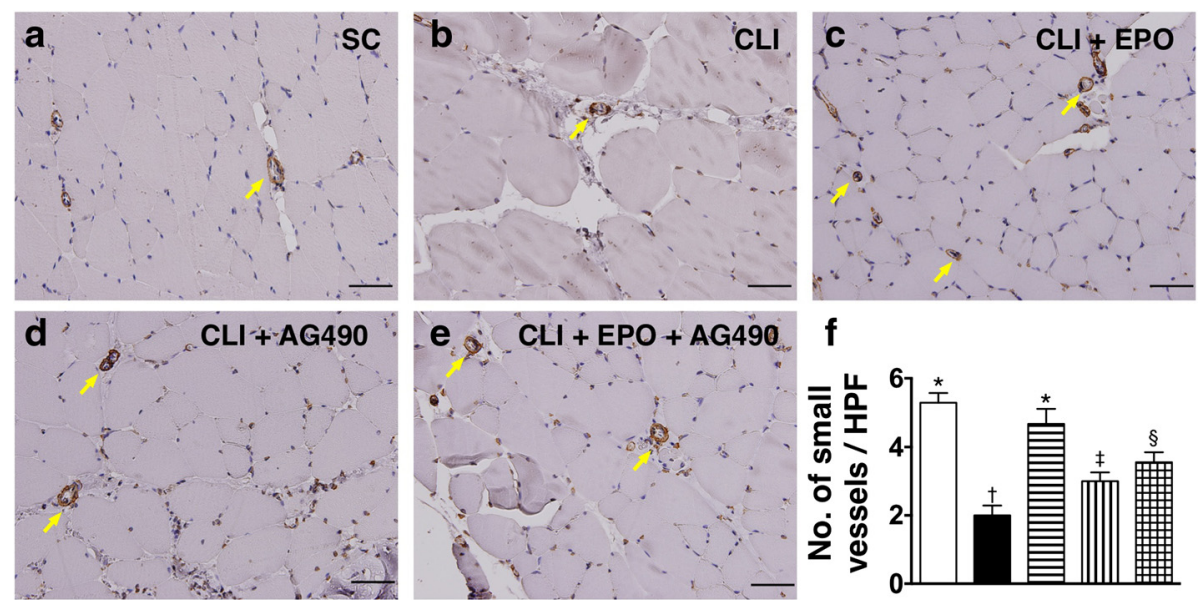

$\square$ Normal $\square \mathrm{CLI}$ Ш

Fig. 8 EPO and AG490 administrations increase the vessel density in quadriceps after critical limb ischemia induction in rats. The distribution of small vessels (diameter $<35 \mu \mathrm{m}$ ) was detected with antibodies against a-smooth muscle actin. a Quadricep section form normal rats. b Quadricep section form rats treated with critical limb ischemia (CLI). c Quadricep section form CLI rats treated with EPO. d Quadricep section form CLI rats treated with AG490. e Quadricep section form CLI rats treated with EPO and AG490. f Quantitation of number of small vessels in quadriceps. Yellow arrows indicate small vessels. Statistical analysis using one-way ANOVA, followed by Bonferroni multiple comparison post hoc test $(n=8$ for each group). Symbols $(*,+, \neq, \S)$ indicate significance at $p$ value less than 0.05

and also reduced the gene expressions of inflammatory factors (Fig. 4). It is worth noting that the combined EPO and AG490 treatment showed synergistic effects in enhancing anti-oxidation and anti-inflammation. At day 14 after CLI, we found that EPO, AG490 and combined EPO and AG490 therapies all increased the blood flow recovery after CLI (Figs. 7 and 8). In addition, results from Western blots showed that the activation of JNK and ERK pathways in quadriceps were regulated by ischemic stress, EPO, and AG490 (Fig. 5).

\section{EPO-mediated intracellular signaling in ischemic limbs}

Among STAT transcription factors, STAT5 is the most prominent substrate to be phosphorylated with JAK2 $[39,40]$, while phosphorylated STAT3 and STAT1 are only found in certain kinds of cells [41, 42]. In the present study, we noted that the phosphorylation of STAT5 and STAT1, but not STAT3, were upregulated by EPO and along with the phosphorylation levels of JAK2. In addition to the JAK2/STAT pathways, several mitogen-activated protein kinases (MAPKs) signaling cascades, including ERK, p38, and JNK, have been reported to play roles in delivering ERO/EPOR signals [43-45]. Results in this study also demonstrated that the expression levels and phosphorylation levels of ERK1/2 were upregulated by EPO and reverted with additional AG490 treatment (Fig. 5). Although previous studies considered the activation of PI3K is important for EPOmediated phosphorylation of ERK [46], the activation of Akt, another PI3K kinase substrate, did not increase after EPO treatment in ischemic quadriceps (Fig. 5). Hence, instead of PI3K, other kinase facility, i.e. Ras/Raf, may contribute in the EPO-mediated, JAK2-dependent, activation of MAPKs after CLI. Although tyrosine phosphorylation of STATs with JAK kinase is found in most cells, some reports demonstrated the existence of JAK2independent phosphorylation of STAT5 [47, 48]. To clarify this issue, we combined EPO and AG490 to treat rat CLI and found that STAT5 activation in ischemic quadriceps was in a JAK2-dependent manner. The inhibitory effect of AG490 in JAK2/STAT signaling has been found in tissues with different pathological stresses, including myocardial hypertrophy [49], liver ischemiareperfusion [35], traumatic brain injury [50], and chronic renal disease [51]. In the present study, we further confirmed the effect of AG490 in inhibiting the activation and JAK2 and its downstream phosphorylation of STAT1, STAT3, and STAT5 in the critical limb ischemia model.

\section{Upregulation of anti-inflammatory and anti-oxidative genes by EPO and AG490}

In addition to roles in erythropoiesis, other functions of EPO, including anti-apoptosis and angiogenesis, have also been demonstrated $[22,30]$. In this study, through the CLI rat model, we found that EPO regulated the gene expression levels of inflammatory and anti-oxidative proteins and showed a protective effect against inflammation and oxidation. Of note, AG490 also provided similar antiinflammatory and anti-oxidation effects, despite its roles 
in blockage of JAK2 activity and impairing anti-apoptosis. The distinct role of AG490 may be through inhibition of JAK2, which reduces gene expression of anti-apoptotic factors in endothelial and skeletal muscle cells, and also inhibits inflammatory cytokine release, proliferation, and activation in inflammatory cells. Hence, the distinct physiological roles of AG490 on different kinds of cells may provide contradictory effects in enhancing recovery from ischemic injury.

\section{Combined EPO and AG490 to treat CLI}

Although the anti-inflammatory capacity of AG490 reduced the generation of inflammatory factors and also increased the expression levels of anti-oxidants, its role in blocking JAK2 activity impaired the anti-apoptotic effects of EPO in the acute phase of CLI. These seemingly contradictory roles may explain the controversy surrounding the effects of AG490 in the treatment of ischemia-reperfusion injury in different organs [35, 52-54]. In this study, the examination of blood flow examination using laser Doppler analysis also showed both EPO and AG490 treatments enhance the blood flow recovery after CLI; however, no additional benefit was found in the combined treatment group. Since JAK2 activity is critical in the function of EPO in preventing ischemia-induced cellular apoptosis, the application of AG490 should be avoided in the acute phase of CLI. Combined EPO and delayed AG490 adminstration may provide better therapeutic efficacy in treating CLI.

\section{Conclusion}

This study indicates that JAK2-dependent STAT activation plays an important role in EPO-mediated enhanced blood flow recovery after CLI induction in rats. Although AG490 inhibits the EPO-induced JAK2/STAT activation in the acute phase of CLI, the benefits of AG490 in anti-inflammation and anti-oxidation still provide a better outcome in rats with CLI. The functional effects of EPO in anti-apoptosis, anti-inflammation, and antioxidation provide therapeutic efficacy for injury recovery after critical limb ischemia.

\section{Acknowledgments \\ The authors thank supports from Chang Gung Medical Research Program Grant (Grant number: CMRPG 8B1221 to HTC; CMRPG 8C1011 and CMRPG 8C1012 to SL) and Ministry of Science and Technology, Taiwan (Grant number MOST-102-2320-B-182A-001-MY3 to SL).}

\section{Authors' contributions}

HTC and SL participated in the design of the study, data acquisition and analysis as well as drafting the manuscript. HTC, CKS, and SYH were responsible for data acquisition, analysis, and interpretation. SL and HKY conceived of the study, and participated in its design and coordination and helped to draft the manuscript. All authors read and approved the final manuscript.

\section{Competing interests}

The authors declare that they have no competing interests.

\section{Ethics approval and consent to participate}

All animal experimental procedures were approved by the Institute of Animal Care and Use Committee of Kaohsiung Chang Gung Memorial Hospital (Approval No. 2012032601) and performed in accordance with the Guide for the Care and Use of Laboratory Animals (NIH publication No. 85-23, National Academy Press, Washington, DC, USA, revised 1996).

\section{Author details}

'Division of Cardiology, Department of Internal Medicine, Kaohsiung Chang Gung Memorial Hospital and Chang Gung University College of Medicine, Kaohsiung, Taiwan. ${ }^{2}$ Institute for Translational Research in Biomedicine, Kaohsiung Chang Gung Memorial Hospital, Kaohsiung, Taiwan. ${ }^{3}$ Center for Shockwave Medicine and Tissue Engineering, Kaohsiung Chang Gung Memorial Hospital, Kaohsiung, Taiwan. ${ }^{4}$ Department of Medical Research, China Medical University Hospital, China Medical University, Taichung, Taiwan. ${ }^{5}$ Department of Nursing, Asia University, Taichung, Taiwan. ${ }^{6}$ Department of Emergency Medicine, E-Da Hospital, I-Shou University, Kaohsiung, Taiwan. 'Department of Anatomy, Graduate Institute of Biomedical Sciences, ,College of Medicine, Chang Gung University, Taoyuan, Taiwan.

Received: 18 January 2016 Accepted: 31 May 2016

Published online: 04 June 2016

\section{References}

1. Shamoun FE, Fankhauser GT, Mookadam M. Vascular medicine: aortic and peripheral arterial disease. Prim Care. 2013;40(1):169-77.

2. Slovut DP, Sullivan TM. Critical limb ischemia: medical and surgical management. Vasc Med. 2008;13(3):281-91.

3. Chung J, Timaran DA, Modrall JG, Ahn C, Timaran CH, Kirkwood ML, Baig MS, Valentine RJ. Optimal medical therapy predicts amputation-free survival in chronic critical limb ischemia. J Vasc Surg. 2013;58(4):972-80.

4. Ortmann J, Gahl B, Diehm N, Dick F, Traupe T, Baumgartner I. Survival benefits of revascularization in patients with critical limb ischemia and renal insufficiency. J Vasc Surg. 2012:56(3):737-745 e731.

5. Simons JP, Schanzer A, Nolan BW, Stone DH, Kalish JA, Cronenwett JL, Goodney PP, Vascular Study Group of New E. Outcomes and practice patterns in patients undergoing lower extremity bypass. J Vasc Surg. 2012; 55(6):1629-36.

6. Gasper WJ, Runge SJ, Owens CD. Management of infrapopliteal peripheral arterial occlusive disease. Curr Treat Options Cardiovasc Med. 2012;14(2): $136-48$

7. Moise MA, Kashyap VS. Treatment of Aortoiliac Occlusive Disease: Medical versus Endovascular versus Surgical Therapy. Curr Treat Options Cardiovasc Med. 2011;13(2):114-28.

8. Dawson DL, Mills JL. Critical limb ischemia. Curr Treat Options Cardiovasc Med. 2007;9(2):159-70.

9. Ramanath V, Gupta D, Jain J, Chaudhary K, Nistala R. Anemia and chronic kidney disease: making sense of the recent trials. Rev Recent Clin Trials. 2012;7(3):187-96.

10. Debeljak N, Sytkowski AJ. Erythropoietin and erythropoiesis stimulating agents. Drug Test Anal. 2012;4(11):805-12.

11. Stickel F, Helbling B, Heim M, Geier A, Hirschi C, Terziroli B, Wehr K, De Gottardi A, Negro F, Gerlach T. Critical review of the use of erythropoietin in the treatment of anaemia during therapy for chronic hepatitis C. J Viral Hepat. 2012;19(2):77-87.

12. Sui X, Krantz SB, Zhao ZJ. Stem cell factor and erythropoietin inhibit apoptosis of human erythroid progenitor cells through different signalling pathways. Br J Haematol. 2000;110(1):63-70.

13. Fan $X$, Heijnen $C$, van der Kooij MA, Groenendaal F, van Bel F. The role and regulation of hypoxia-inducible factor-1alpha expression in brain development and neonatal hypoxic-ischemic brain injury. Brain Res Rev. 2009;62(1):99-108.

14. Haase VH. Regulation of erythropoiesis by hypoxia-inducible factors. Blood Rev. 2013;27(1):41-53.

15. Erbayraktar S, Yilmaz $\mathrm{O}$, Gokmen N, Brines M. Erythropoietin is a multifunctional tissue-protective cytokine. Curr Hematol Rep. 2003;2(6):465-70.

16. Dang J, Jia R, Tu Y, Xiao S, Ding G. Erythropoietin prevents reactive oxygen species generation and renal tubular cell apoptosis at high glucose level. Biomed Pharmacother. 2010;64(10):681-5. 
17. Adelibieke Y, Shimizu H, Saito S, Mironova R, Niwa T. Indoxyl sulfate counteracts endothelial effects of erythropoietin through suppression of Akt phosphorylation. Circ J. 2013;77(5):1326-36.

18. Zhong YS, Yao HP, Deng LF, Cheng Y, Min YJ. Effect of erythropoietin on the survival of retinal neurocytes in culture upon serum withdrawal. Neurosciences. 2010;15(3):152-8.

19. Tramontano AF, Muniyappa R, Black AD, Blendea MC, Cohen I, Deng L, Sowers JR, Cutaia MV, El-Sherif N. Erythropoietin protects cardiac myocytes from hypoxia-induced apoptosis through an Akt-dependent pathway. Biochem Biophys Res Commun. 2003;308(4):990-4.

20. Zhang X, Li QY, Xiao BG. Anti-inflammatory effect of erythropoietin therapy on experimental autoimmune encephalomyelitis. Int J Neurosci. 2012;122(5): 255-62.

21. Ribatti D. Erythropoietin and tumor angiogenesis. Stem Cells Dev. 2010; 19(1):1-4.

22. Hou X, Wu X, Ma J, Lv X, Jin X. Erythropoietin augments the efficacy of therapeutic angiogenesis induced by allogenic bone marrow stromal cells in a rat model of limb ischemia. Mol Biol Rep. 2010;37(3):1467-75.

23. Zhang Y, Wang L, Dey S, Alnaeeli M, Suresh S, Rogers H, Teng R, Noguchi $C T$. Erythropoietin action in stress response, tissue maintenance and metabolism. Int J Mol Sci. 2014;15(6):10296-333.

24. Gut N, Piecha G, Aldebssi F, Schaefer S, Bekeredjian R, Schirmacher P, Ritz E, Gross-Weissmann ML. Erythropoietin combined with ACE inhibitor prevents heart remodeling in 5/6 nephrectomized rats independently of blood pressure and kidney function. Am J Nephrol. 2013;38(2):124-35.

25. Sepodes B, Maio R, Pinto R, Sharples E, Oliveira P, McDonald M, Yaqoob M, Thiemermann C, Mota-Filipe H. Recombinant human erythropoietin protects the liver from hepatic ischemia-reperfusion injury in the rat. Transpl Int. 2006;19(11):919-26.

26. Lipsic E, van der Meer $\mathrm{P}$, Henning RH, Suurmeijer AJ, Boddeus KM, van Veldhuisen DJ, van Gilst WH, Schoemaker RG. Timing of erythropoietin treatment for cardioprotection in ischemia/reperfusion. J Cardiovasc Pharmacol. 2004;44(4):473-9.

27. Yip HK, Tsai TH, Lin HS, Chen SF, Sun CK, Leu S, Yuen CM, Tan TY, Lan MY, Liou CW, et al. Effect of erythropoietin on level of circulating endothelial progenitor cells and outcome in patients after acute ischemic stroke. Crit Care. 2011;15(1):R40

28. Chua S, Leu S, Lin YC, Sheu JJ, Sun CK, Chung SY, Chai HT, Lee FY, Kao YH, Wu CJ, et al. Early erythropoietin therapy attenuates remodeling and preserves function of left ventricle in porcine myocardial infarction. J Invest Med. 2011;59(3):574-86.

29. Lacombe C, Mayeux P. The molecular biology of erythropoietin. Nephrol Dial Transplant. 1999:14 Suppl 2:22-8.

30. Bittorf $T$, Jaster R, Ludtke B, Kamper B, Brock J. Requirement for JAK2 in erythropoietin-induced signalling pathways. Cell Signal. 1997;9(1):85-9.

31. Huang C, Ma R, Sun S, Wei G, Fang Y, Liu R, Li G. JAK2-STAT3 signaling pathway mediates thrombin-induced proinflammatory actions of microglia in vitro. J Neuroimmunol. 2008:204(1-2):118-25.

32. Kandalam U, Clark MA. Angiotensin II activates JAK2/STAT3 pathway and induces interleukin- 6 production in cultured rat brainstem astrocytes. Regul Pept. 2010;159(1-3):110-6.

33. Yang $X$, He G, Hao Y, Chen C, Li M, Wang Y, Zhang G, Yu Z. The role of the JAK2-STAT3 pathway in pro-inflammatory responses of EMF-stimulated N9 microglial cells. J Neuroinflammation. 2010;7:54.

34. Park JS, Lee J, Lim MA, Kim EK, Kim SM, Ryu JG, Lee JH, Kwok SK, Park KS, Kim HY, et al. JAK2-STAT3 blockade by AG490 suppresses autoimmune arthritis in mice via reciprocal regulation of regulatory T Cells and Th17 cells. J Immunol. 2014;192(9):4417-24.

35. Freitas MC, Uchida Y, Zhao D, Ke B, Busuttil RW, Kupiec-Weglinski JW. Blockade of Janus kinase-2 signaling ameliorates mouse liver damage due to ischemia and reperfusion. Liver Transpl. 2010;16(5):600-10.

36. Leu S, Lu HI, Sun CK, Sheu JJ, Chen YL, Tsai TH, Yeh KH, Chai HT, Chua S, Tsai $C Y$, et al. Retention of endothelial progenitor cells in bone marrow in a murine model of endogenous tissue plasminogen activator (tPA) deficiency in response to critical limb ischemia. Int J Cardiol. 2014;170(3):394-405.

37. Sun CK, Leu S, Sheu JJ, Tsai TH, Sung HC, Chen YL, Chung SY, Ko SF, Chang HW, Yip HK. Paradoxical impairment of angiogenesis, endothelial function and circulating number of endothelial progenitor cells in DPP4-deficient rat after critical limb ischemia. Stem Cell Res Ther. 2013;4(2):31.
38. Zhande R, Karsan A. Erythropoietin promotes survival of primary human endothelial cells through PI3K-dependent, NF-kappaB-independent upregulation of BCl-xL. Am J Physiol Heart Circ Physiol. 2007;292(5):H2467-74.

39. Bittorf T, Seiler J, Ludtke B, Buchse T, Jaster R, Brock J. Activation of STAT5 during EPO-directed suppression of apoptosis. Cell Signal. 2000;12(1):23-30.

40. Sawyer ST, Penta K. Association of JAK2 and STAT5 with erythropoietin receptors. Role of receptor phosphorylation in erythropoietin signal transduction. J Biol Chem. 1996;271(50):32430-7.

41. Yamamura $Y$, Senda $H$, Noda M, Ikawa Y. Activation of the JAK1-STAT5 pathway by binding of the Friend virus gp55 glycoprotein to the erythropoietin receptor. Leukemia. 1997;11 Suppl 3:432-4.

42. Gao W, McCormick J, Connolly M, Balogh E, Veale DJ, Fearon U. Hypoxia and STAT3 signalling interactions regulate pro-inflammatory pathways in rheumatoid arthritis. Ann Rheum Dis. 2015;74:1275-83.

43. Lee SM, Nguyen TH, Park MH, Kim KS, Cho KJ, Moon DC, Kim HY, Yoon DY, Hong JT. EPO receptor-mediated ERK kinase and NF-kappaB activation in erythropoietin-promoted differentiation of astrocytes. Biochem Biophys Res Commun. 2004;320(4):1087-95.

44. Chong ZZ, Lin SH, Kang JQ, Maiese K. Erythropoietin prevents early and late neuronal demise through modulation of Akt1 and induction of caspase 1, 3 , and 8. J Neurosci Res. 2003;71(5):659-69.

45. Shan R, Price JO, Gaarde WA, Monia BP, Krantz SB, Zhao ZJ. Distinct roles of JNKs/p38 MAP kinase and ERKs in apoptosis and survival of HCD-57 cells induced by withdrawal or addition of erythropoietin. Blood. 1999:94(12): 4067-76.

46. Schmidt EK, Fichelson S, Feller SM. PI3 kinase is important for Ras, MEK and Erk activation of Epo-stimulated human erythroid progenitors. BMC Biol. 2004;2:7.

47. Mannello F, Tonti GA. Erythropoietin and its receptor in breast cancer: putting together the pieces of the puzzle. Oncologist. 2008;13(7):761-8.

48. Tsuji-Takayama K, Otani T, Inoue T, Nakamura S, Motoda R, Kibata M, Orita K. Erythropoietin induces sustained phosphorylation of STAT5 in primitive but not definitive erythrocytes generated from mouse embryonic stem cells. Exp Hematol. 2006;34(10):1323-32.

49. Manukyan I, Galatioto J, Mascareno E, Bhaduri S, Siddiqui MA. Cross-talk between calcineurin/NFAT and Jak/STAT signalling induces cardioprotective alphaB-crystallin gene expression in response to hypertrophic stimuli. J Cell Mol Med. 2010;14(6B):1707-16.

50. Zhao JB, Zhang Y, Li GZ, Su XF, Hang CH. Activation of JAK2/STAT pathway in cerebral cortex after experimental traumatic brain injury of rats. Neurosci Lett. 2011;498(2):147-52.

51. Li R, Yang N, Zhang L, Huang Y, Zhang R, Wang F, Luo M, Liang Y, Yu X. Inhibition of Jak/STAT signaling ameliorates mice experimental nephrotic syndrome. Am J Nephrol. 2007;27(6):580-9.

52. Clarke C, Sakai N, Tevar AD, Schuster R, Edwards MJ, Lentsch AB. STAT3 does not regulate acute liver injury after ischemia/reperfusion. J Surg Res. 2011;171(2):814-8

53. Cheppudira BP, Girard BM, Malley SE, Dattilio A, Schutz KC, May V, Vizzard MA. Involvement of JAK-STAT signaling/function after cyclophosphamideinduced bladder inflammation in female rats. Am J Physiol Renal Physiol. 2009;297(4):F1038-44.

54. Chattong S, Tanamai J, Kiatsomchai P, Nakatsu M, Sereemaspun A, Pimpha N, Praditpornsilpa K, Rojanathanes R, Sethpakadee A, Tungsanga K, et al. Glutaraldehyde erythropoietin protects kidney in ischaemia/reperfusion injury without increasing red blood cell production. Br J Pharmacol. 2013; 168(1):189-99. 